\title{
Examen Neurológico en el Equino de Deporte
}

\author{
Cristóbal Dörner SM${ }^{1}$., Adolfo Godoy $\mathbf{P}^{1}$. \\ ${ }^{1}$ Facultad de Ciencias Veterinarias y Pecuarias. Universidad de Chile. Casilla 2 Correo 15, La \\ Granja. Santiago, Chile. agodoy@uchile.cl
}

\begin{abstract}
Resumen
El veterinario clínico se enfrenta frecuentemente al desafío de tener que diagnosticar trastornos neurológicos en el caballo de deporte. En ocasiones, las neuropatías muestran signos clínicos poco claros y confusos, y requieren de un diagnóstico diferencial exhaustivo con otros sistemas del organismo. Existe la creencia que el examen del sistema neurológico en el equino es un proceso difícil y tedioso, sin embargo éste es un procedimiento relativamente sencillo.

El Sistema Nervioso se clasifica desde un punto de vista anatómico en Sistema Nervioso Central, que incluye encéfalo y médula espinal, y Sistema Nervioso Periférico, formado por los nervios que salen desde la médula espinal y los pares craneales que salen del encéfalo.

El examen del sistema nervioso debe ser sistemático y acucioso. Debe comenzar desde la cabeza e ir avanzando hasta llegar a la cola. La evaluación del ejemplar va a comenzar con la observación general del paciente, incluyendo la actitud y su interacción con el medio, posición del cuerpo, cabeza y miembros así como la simetría del desarrollo muscular. Los componentes neurológicos que se evalúan comúnmente, son los nervios craneales, respuestas posturales de los miembros, análisis del paso y la evaluación de la cola y ano. Al evaluar la cabeza, lo ideal es valorar el comportamiento o conducta, estado mental, posición y movimiento de la cabeza y cuello.

La marcha da una visión general de la función del tronco encefálico, cerebelo, médula espinal, nervios periféricos y músculos. Lo principal que se debe evaluar neurológicamente es debilidad y ataxia. El animal se debe examinar mientras camina, trota, gira y se desplaza hacia atrás. Muchas veces los signos son sutiles por lo que se si se puede, se debe observar a animal subiendo y/o bajando una pendiente, con el cuello extendido, corriendo a campo libre y caminando con los ojos vendados; con estas maniobras los signos debieran hacerse más evidentes.
\end{abstract}

Palabras claves: Sistema Nervioso Central, Sistema Nervioso Periférico, Neuropatías, Equinos.

\section{Introducción}

Las patologías del sistema nervioso en el equino de deporte constituyen entidades clínicas frecuentes si se consideran aquellas alteraciones que comprometen al sistema nervioso en forma primaria, como aquellas que ocurriendo la alteración a otro nivel del organismo repercuten en éste llevando a neuropatías de origen secundario. Muchas de estas enfermedades muestran signos clínicos poco claros y confusos, lo que hace necesario un diagnóstico diferencial exhaustivo (Palencia, 2007).

Existe la creencia que el examen del sistema neurológico en el equino es un proceso difícil y tedioso, sin embargo, al realizarse en forma adecuada se constituye en un procedimiento relativamente sencillo. Para su evaluación, el médico veterinario debe poseer obviamente el conocimiento básico sobre neuroanatomía como también de los desordenes neurológicos que 
pueden afectar a esta especie (Palencia, 2007; Furr y Reed, 2008).

Resulta de gran importancia para el éxito del diagnóstico, un abordaje sistemático, ordenado y acucioso del caballo. Se debe comenzar desde la cabeza e ir avanzando hacia caudal hasta llegar a la cola (Reed et al, 2004). Al sospechar de una patología de carácter neurológico, no debe dejarse de lado la completa revisión física del paciente, con el objeto de dilucidar si la sintomatología nerviosa que presenta el ejemplar corresponde efectivamente a un problema neurológico y no que el sistema nervioso este sólo actuando como un medio para expresar alteraciones que están ocurriendo a otro nivel. (hígado, riñón), (Orsini y Divers, 2000; Reed et al, 2004; Mazan, 2010). Si bien es cierto, el abordaje neurológico no es complicado, la interpretación de los resultados requiere cierto grado de experiencia y adecuada observación por parte del veterinario (Grant, 2010).

Como médicos veterinarios, nuestros principales objetivos frente a un paciente es lograr dilucidar si la anormalidad observada corresponde o no a una alteración de carácter neurológico y al mismo tiempo "neurolocalizar" el origen de ésta (Colahan et al, 1998.; Reed et al, 2004).

La evaluación del ejemplar debe comenzar con la observación general del paciente, incluyendo la actitud y su interacción con el medio, posición del cuerpo, cabeza y miembros así como la simetría del desarrollo muscular (Furr y Reed, 2008). Los componentes neurológicos que se evalúan comúnmente, son los nervios craneales, respuestas posturales de los miembros, análisis del paso y la evaluación de la cola y ano.

El objetivo de este trabajo es presentar una actualización sobre conceptos de neuroanatomía, como también discutir el adecuado procedimiento para evaluar e interpretar las alteraciones que afectan al sistema nervioso en el equino de deporte.

\subsection{Conceptos básicos de neuroanatomía}

El Sistema Nervioso se clasifica desde un punto de vista anatómico en Sistema Nervioso Central, SNC, que incluye el encéfalo (cerebro, cerebelo, bulbo raquídeo y tubérculos cuadrigéminos) y médula espinal, y Sistema Nervioso Periférico,
SNP, formado por los nervios que salen desde la médula espinal y los pares craneales que salen del encéfalo. El S.N.P., se clasifica desde un punto de vista funcional en Sistema Nervioso Somático responsable del control de la musculatura estriada y Sistema Nervioso Vegetativo o autónomo, dividido en Sistema Nervioso Simpático y Sistema Nervioso Parasimpático (Radostits et al, 2002; Palencia, 2007), que regulan el control visceral y la musculatura involuntaria. (Sandoval, 2009). Así se habla de lesiones centrales, cuando las disfunciones neurológicas tienen su origen en el SNC, y lesiones periféricas, cuando estas se localizan en el SNP (Colahan et al, 1998).

El encéfalo se aloja en el interior del cráneo, en el cual podemos distinguir los hemisferios cerebrales, el cerebelo y el tronco encefálico (Radostits et al, 2002). Este último da origen a la médula espinal, ubicada y protegida por la columna vertebral. Estos elementos están formados por numerosas estructuras de procesamiento de la información sensitiva (los núcleos de las neuronas ubicadas en la sustancia gris), y al mismo tiempo, por tractos de fibras nerviosas (axones de las neuronas), que transportan la información a los centros de procesamiento y conectan unas estructuras con otras (formando la sustancia blanca) (Palencia, 2007).

El Sistema Nervioso Periférico (SNP) se encuentra constituido por una gran cantidad de nervios, que funcionalmente se clasifican en dos tipos (Palencia, 2007): fibras aferentes, que "transportan" la información sensitiva a la médula y encéfalo, y fibras eferentes que emiten la información motora desde los centros superiores y médula hacia los músculos y órganos. Algunos nervios salen directamente del encéfalo, inervando principalmente la cabeza, son los nervios craneales. Los nervios que salen de la médula son los nervios espinales (Guyton y Hall, 2001; Palencia, 2007).

El Sistema Nervioso tiene como función, recoger la información sensitiva de todo el organismo, a través de los nervios periféricos, y llevarla hasta médula y encéfalo, lugares en los cuales dicha información va a ser procesada para que consecuentemente se produzca una respuesta a esos estímulos enviando señales a los órganos efectores, para obtener una respuesta motora (Guyton y Hall, 2001; Palencia, 2007). La 
neurona aferente, sensitiva, transporta un impulso nervioso desde los diferentes receptores sensitivos (receptores de propiocepción, presión, dolor, etc.) hasta médula, donde hace sinapsis con una segunda neurona eferente, motora, para desencadenar la respuesta motora. A esta unidad básica, de una respuesta motora a un estimulo sensitivo, a nivel medular, la denominamos arco reflejo (Palencia, 2007, Mayhew, 2009a). La información sensitiva viaja a los centros superiores del encéfalo, y la respuesta motora, se realiza a dos niveles; en primer lugar en una neurona motora superior que viaja por el encéfalo y médula y hace sinapsis con las interneuronas en el asta ventral de la sustancia gris de la médula, para volver a hacer sinapsis con la neurona motora inferior que lleva la información hasta los músculos. Por ello, definimos el Sistema Motor Eferente como el formado por la Neurona Motora Superior, NMS, ubicada en el SNC, y la Neurona Motora Inferior, NMI, cuyo soma neuronal se encuentra en el asta ventral de la sustancia gris de la médula espinal, y los axones que se proyectan sobre los músculos estriados (Reed et al, 2004; Palencia, 2007; Furr y Reed, 2008; Dörner, 2009). Por otro lado, la inervación simpática y parasimpática de la misma estructura es usualmente antagónica (Sandoval, 2009). El sistema nervioso simpático prepara al cuerpo para la clásica respuesta de "pelear o escapar" ("fight or flight”). La inervación parasimpática promueve las funciones corporales de "descanso y recuperación” (Masty, 2008; Sandoval, 2009).

Cuando estamos frente a un déficit o debilidad de la MNS producido por algún tipo de lesión, nos vamos a encontrar con un paciente que presenta espasticidad, aumento del tono, respuesta refleja exagerada, leve atrofia muscular y no existe presencia de fasciculaciones. Por otro lado, cuando se ve afectada la MNI, el paciente va a presentar flacidez, disminución del tono, disminución de la respuesta refleja, gran atrofia muscular y presencia de fasciculaciones (Furr y Reed, 2008; Mayhew, 2008; Dörner, 2009; Mayhew, 2009a). La valoración de los reflejos, es decir la respuesta refleja a los diferentes estímulos provocados en el animal, tanto en los nervios craneales como en los nervios espinales, es una de las pruebas en que se basa el examen clínico neurológico, por el cual el veterinario clínico confirma la alteración neurológica y la localización neuroanatómica de la lesión. Por ello se debe de incorporar en la exploración general del caballo, pruebas clínicas neurológicas básicas, que confirmen o no la sospecha de lesión neurológica y que justifiquen un examen neurológico más exhaustivo (Mackay y Mayhew, 1998; Reed et al, 2004; Furr y Reed, 2008).

Los 12 pares o nervios craneanos son los siguientes: Olfatorio (I); Ocular (II); Oculomotor (III); Troclear (IV); Trigémino (V); Abducen (VI); Facial (VII); Vestibulococlear (VIII); Glosofaríngeo (IX); Vago (X); Espinal accesorio (XI) ;Hipogloso (XII) (Colahan et al, 1998; Budras et al, 2002; Furr y Reed, 2008).

\subsection{Examen Neurologico}

El examen neurológico debe realizarse a partir de una sospecha de alguna alteración nerviosa detectada en el examen físico. El examen del sistema nervioso debe ser sistemático y acucioso. Debe comenzar desde la cabeza e ir avanzando hasta llegar a la cola. Lo que se debe evaluar es: (Colahan et al, 1998; Dörner, 2009):

1. Cabeza

- Conducta

- Estado mental

- Postura y coordinación de la cabeza

- Nervios craneales

2. Marcha y postura

3. Cuello y miembros anteriores

4. Dorso y miembros posteriores

5. Cola y ano

\section{Examen De La Cabeza}

La evaluación de la conducta debe ir de la mano con los antecedentes anamnesicos entregados por el dueño para averiguar cuál es la conducta normal y habitual del animal. En lo posible hay que tratar en una primera aproximación al paciente que esta sea una observación desde lejos, de manera de no inducir con nuestra presencia conductas o reacciones por parte del animal que nos lleven a confundir la interpretación de esta. (Radostits et al, 2002; Mayhew, 2009b). Lo ideal es evaluar el comportamiento o conducta, estado mental, posición y movimiento de la cabeza y cuello (Mayhew, 2009b). Cambios de conducta serían: Ejercer presión de la cabeza contra objetos, andar aberrante compulsivo; marcha en círculos, lamido a objetos y agresión, entre otros (Reed et al, 2004; Furr y Reed, 2008). . 
Estas conductas indican enfermedad cerebral. Generalmente son simétricas, pero en el caso de presentarse unilateralmente, la marcha en círculos compulsiva tiende a ser hacia el lado donde se encuentra la lesión y coloca la cabeza y cuello inclinados en esa misma dirección (Mackay y Mayhew, 1998).

\section{Estado Mental}

Se refiere al estado de conciencia y por lo tanto se debe evaluar la respuesta a estímulos visuales, táctiles, auditivos, dolorosos, olfatorios y gustativos.

Los estados que podemos encontrar son: estupor, somnolencia, depresión, letargia, delirio, semi coma y coma (Radostits et al, 2002; Mackay 2010c). Cuando un caballo se encuentra letárgico, lo vamos a pesquisar porque ha perdido su expresión facial y se observa una moderada caída de las orejas y párpados, mientras que su respuesta a los estímulos externos es lenta. El estupor se caracteriza porque el ejemplar se encuentra en estación con la cabeza agachada y solo responde a estímulos externos fuertes (Mackay, 2010c). Caballos que se encuentran en decúbito y aparentemente en "estupor" son aquellos que se encuentran en un estado de semi coma. El coma se caracteriza por una falta de respuesta completa a los estímulos normales (Colahan et al, 1998; Furr y Reed, 2008; Mackay, 2010c).

Alguna alteración del estado de conciencia, denota afección del sistema reticular activante del tronco encefálico y de los hemisferios cerebrales.

\section{Postura y Coordinación}

Para evaluar la orientación de la cabeza, cuando se realiza la observación del ejemplar desde el frente, se debe dilucidar algún tipo de balanceo o inclinación de la cabeza (Orsini y Divers, 2000; Mackay, 2010d). Las lesiones vestibulares producen inclinación de la cabeza hacia el lado de la lesión y también marcha en círculo. Se caracteriza por una desviación de la nuca hacia lateral, pero el cuello y el hocico se mantienen en la línea media (; Mackay, 2010c; Mackay, 2010d). $\mathrm{Si}$ la afección es bilateral, los ejemplares presentan un amplio movimiento de columpio de la cabeza (Colahan et al, 1998).
Cuando observamos la cabeza desde lateral, hay que evaluar si es que la cabeza se encuentra más debajo de lo normal, podemos sospechar de una debilidad muscular ya sea neurológica o no. Se ha descrito que cuando el cuello se encuentra extendido, se puede deber a problemas cervicales (Stashak, 2002) o a alguna afección de las bolsas guturales (Mackay, 2010c; Mackay, 2010d).

También se puede evaluar la funcionalidad cerebelar, la que produce un movimiento aparentemente espasmódico, también llamado "tremor de intención" cuando ésta se ve afectada (Reed et al, 2004; Mackay, 2010c; Mackay, 2010d). El cerebelo es también el que controla los movimientos finos de la cabeza y extremidades.

\section{Nervios Craneales}

- Olfatorio (I): Es raro que se afecte. Evaluación: capacidad del ejemplar para oler su alimento o la mano del veterinario (Colahan et al, 1998).

- Ocular (II): Su alteración produce ceguera. Se evalúa mediante la respuesta a la amenaza (Foto. $\mathrm{N}^{\mathrm{o}} 1$ ). Esta prueba debe realizarse parado en frente del caballo, abriendo la palma de la mano provocando un gesto intimidante hacia el ojo del caballo. Esta respuesta debe ser realizada en ambos ojos y en dirección nasal y temporal (Furr y Reed, 2008; Mackay, 2010c). La respuesta normal se establece como el cierre de los parpados y/o la cabeza puede efectuar un movimiento de rechazo (Colahan et al, 1998).

- Oculomotor (III): se evalúa mediante el diámetro pupilar y respuesta a la luz. La estimulación lumínica de un ojo, debe entregar como respuesta la constricción de ambas pupilas. Una afección de este nervio, se presentará con pupilas midriáticas o anisocoria dependiendo si es uni o bilateral. Por otro lado también se produciría un estrabismo ventro lateral con la parálisis de este nervio (Mackay y Mayhew, 1998; Furr y Reed, 2008).

- Troclear (IV): La parálisis del nervio troclear produce un estrabismo dorso medial (Mackay y Mayhew, 1998; Furr y Reed, 2008).

- Trigémino (V): Daño bilateral de su rama motora produce la caída de la mandíbula e imposibilidad para masticar. Por otro lado, el daño de sus fibras sensitivas se traduce con la pérdida 
sensitiva de la cara, no existiendo respuesta refleja de las orejas, ojos, ollares y labios con leves pinchazos (Mackay, 2010d; Reed et al, 2004).

- Abducen (VI): El daño de este nervio provoca un estrabismo medial y el reflejo corneal se encontraría disminuido (globo ocular se retrae menos y parpados se mantienen abiertos) (Colahan et al, 1998; Furr y Reed, 2008).

- Facial (VII): El nervio facial se distribuye en los músculos relacionados con la expresión facial. Se evalúa con reflejos que inducen el cierre de los parpados (amenaza, palpebral y corneal) (Foto. $\mathrm{N}^{\circ}$ 1) y el movimiento de las orejas, labios y nariz. Caída de la oreja, del labio superior y ptosis del párpado superior son signos de parálisis facial (Colahan et al, 1998; Furr y Reed, 2008). El componente sensitivo del nervio facial se distribuye en la lengua y en el oído medio (Mackay, 2010d).

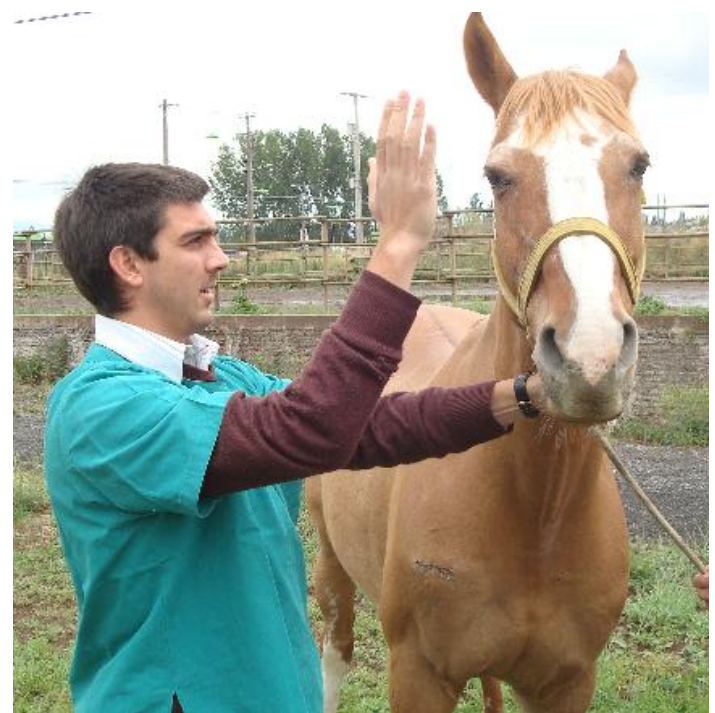

Foto $\mathrm{N}^{\circ}$ 1. Evaluación neurológica, mediante la respuesta a la amenaza.

- Vestibulococlear (VIII): El sistema vestibular controla los movimientos del ojo durante el movimiento de la cabeza y es el responsable de la orientación del caballo en relación a la gravedad (Mackay, 2010d). La cabeza debe ser examinada en busca de inclinación de ella hacia el lado de la lesión. También se debe evaluar la marcha, buscando marcha en círculo. La afección del sistema vestibular puede ser central o local. Una afección central resulta en nistagmos que varia con el posicionamiento de la cabeza (Furr y Reed,
2008). Una afección bilateral se traduce en sordera, siendo muy difícil de diagnosticar.

- Glosofaríngeo (IX): El noveno par inerva la faringe y laringe, por lo tanto, se debe evaluar mediante los sonidos normales de la laringe y por el reflejo de deglución. Los signos más relevantes son la parálisis faríngea y laríngea. La disfunción de este nervio puede ser evaluada mediante el posicionamiento de una sonda naso gástrica buscando una correcta deglución ((Mackay y Mayhew, 1998; Furr y Reed, 2008). La función laríngea se evalúa con la respuesta aductora laríngea esta prueba debe hacerse mientras se observa la laringe con endoscopio y se aplica un pequeño golpe caudal a la escápula lo que debiese producir una aducción contralateral del cartílago aritenoides (Palencia, 2007; Furr y Reed, 2008). El gusto, además de ser captado por el nervio facial en los 2/3 rostrales de la lengua, también es posible encontrar papilas gustativas en los labios, paladar blando y faringe (Mackay, 2010d). El componente sensitivo del nervio glosofaríngeo se encuentra en el 1/3 posterior de la lengua.

- Vago (X): El decimo par, al igual que el nervio glosofaríngeo, tiene un componente motor y sensitivo e inerva la faringe y laringe (Colahan et al, 1998), por lo tanto, se debe evaluar mediante los sonidos normales de la laringe y por el reflejo de deglución. Los signos más relevantes son la parálisis faríngea y laríngea. La disfunción de este nervio puede ser evaluada mediante el posicionamiento de una sonda naso gástrica buscando una correcta deglución (Mackay y Mayhew, 1998; Furr y Reed, 2008). La función laríngea se evalúa de igual forma para lo descrito en caso del nervio Glosofaringeo con un pequeño golpe detrás de la escapula lo cual induce aducción de la laringe, esta prueba debe hacerse mientras se observa la laringe con endoscopio (Palencia, 2007; Furr y Reed, 2008). El componente sensitivo se distribuye en la faringe y el paladar blando (Mackay, 2010d)

- Espinal accesorio (XI): Provee la inervación motora del músculo trapecio y la porción craneal del esternocefálico. Detección mediante electromiografía (Mackay y Mayhew, 1998).

- Hipogloso (XII): Se evalúa inspeccionando la lengua en busca de simetría, movimientos normales y atrofia. En general la lengua se encuentra protruida afectando la prehensión y 
deglución cuando existe afección en el nervio hipogloso bilateralmente, pero cuando es unilateral se observa atrofia unilateral de la lengua, retracción débil pero no suele permanecer protruida fuera de la boca (Mackay y Mayhew, 1998).

Para diagnosticar y diferenciar si el compromiso es a nivel central (tronco encefálico) o a nivel de los nervios periféricos, se debe identificar el compromiso de estructuras de la médula oblonga, en donde se produce depresión, ataxia, además de los signos del nervio craneal afectado.

\section{Evaluación de la Marcha y la Postura}

La marcha da una visión general de la función del tronco encefálico, cerebelo, la médula espinal, los nervios periférico y los músculos. Lo principal que se debe evaluar neurológicamente es debilidad y ataxia (Mazan, 2010). El animal se debe evaluar mientras camina, trota, gira o camina hacia atrás. Muchas veces los signos son sutiles por lo que se si se puede, se debe evaluar a animal subiendo y/o bajando una pendiente, con el cuello extendido, corriendo a campo libre y caminando con los ojos vendados; asi con estas maniobras como se discutió anteriormente los signos debiesen incrementarse (Grant, 2010; Mazan, 2010, Mazan 2010b).

DEBILIDAD O PARESIA: En general se manifiesta con el arrastre de los miembros o disminución del arco de elevación del paso. Se evalúa con la marcha en círculos, en donde le caballo puede caer si carga el peso sobre el miembro débil. La debilidad de los miembros pélvicos se evalúa tirando la cola lateralmente cuando el caballo camina y así se determina la resistencia que ofrece cada miembro (Grant, 2010; Mazan, 2010, Mazan 2010b). La debilidad de un miembro se pesquisa ya que el animal evidencia temblores. En el caso de presentarse debilidad de los cuatro miembros pero sin ataxia, hay que considerar enfermedad neuromuscular (Colahan et al, 1998)

ATAXIA: La ataxia se presenta cuando existe una alteración en la propiocepción. La propiocepción es posible debido a una red de receptores en músculos, tendones y articulaciones, los cuales posibilitan tener el control y el conocimiento de los miembros en el espacio (Furr y Reed, 2008). Las vías propioceptivas se encuentran en múltiples nervios sensitivos que van a pasar por la medula espinal, conformando el tracto espinocerebelar (Guyton y Hall, 2001; Furr y Reed, 2008). Otras vías propioceptivas pasan por la medula espinal hasta llegar a los núcleos de relevo presentes en la corteza sensorial somática (Furr y Reed, 2008). Este déficit propioceptivo puede ser consciente o inconsciente dependiendo de que si su integración es en la corteza cerebral o en el cerebelo respectivamente (Guyton y Hall, 2001). Generalmente se exacerba al caminar en una loma o con la cabeza elevada. También puede ser más pronunciada si el animal trota o galopa libremente. Cuando el ejemplar gira en círculos, en condiciones normales el caballo realiza una secuencia de movimientos en varios tiempos (Foto. $\mathrm{N}^{\circ} 2$ ); una primera flexión lateral cervical hacia el lado girado, que debe de ser un movimiento suave que refleje flexibilidad y facilidad de movimiento de la musculatura cervical (en caso contrario girara todo el cuerpo por incapacidad de flexión del cuello) (Grant, 2010; Mazan, 2010, Mazan 2010b). A continuación el animal realizará un movimiento donde hará un cruzamiento de la extremidad anterior exterior por delante de la extremidad interior, mientras la extremidad posterior interior cruza por delante de la exterior, en una secuencia de movimientos suaves, constantes y sin mostrar signos de pivotamiento, arrastre o posicionamiento aleatorio de las extremidades, que reflejaría una incoordinación o ataxia, en la mayoría de los casos por mielopatías compresivas a nivel cervical (Mackay y Mayhew, 1998).

HIPOMETRÍA: Disminución de la intensidad del paso. Hay muy poca flexión de los miembros, en especial del carpo y del tarso. También ha sido definido como espasticidad o rigidez en la marcha, asemejando una marcha envarada (Colahan et al, 1998). Es más fácil observar una marcha hipométrica cuando el animal se hace caminar hacia atrás o en una pendiente (Mackay y Mayhew, 1998). En general indica una lesión de la motoneurona superior o del sistema vestibular (Mackay y Mayhew, 1998, Mackay, 2010a).

HIPERMETRÍA: Aumento de la intensidad del paso. Hay un exceso de la flexión de las articulaciones lo que puede resultar en un paso más largo o más corto. Es una presentación típica de enfermedades cerebelares pero también puede deberse a alteración de los nervios periféricos como en el caso del "Stringhalt" (Colahan et al, 1998; Stashak, 2002; Mackay, 2010b). 


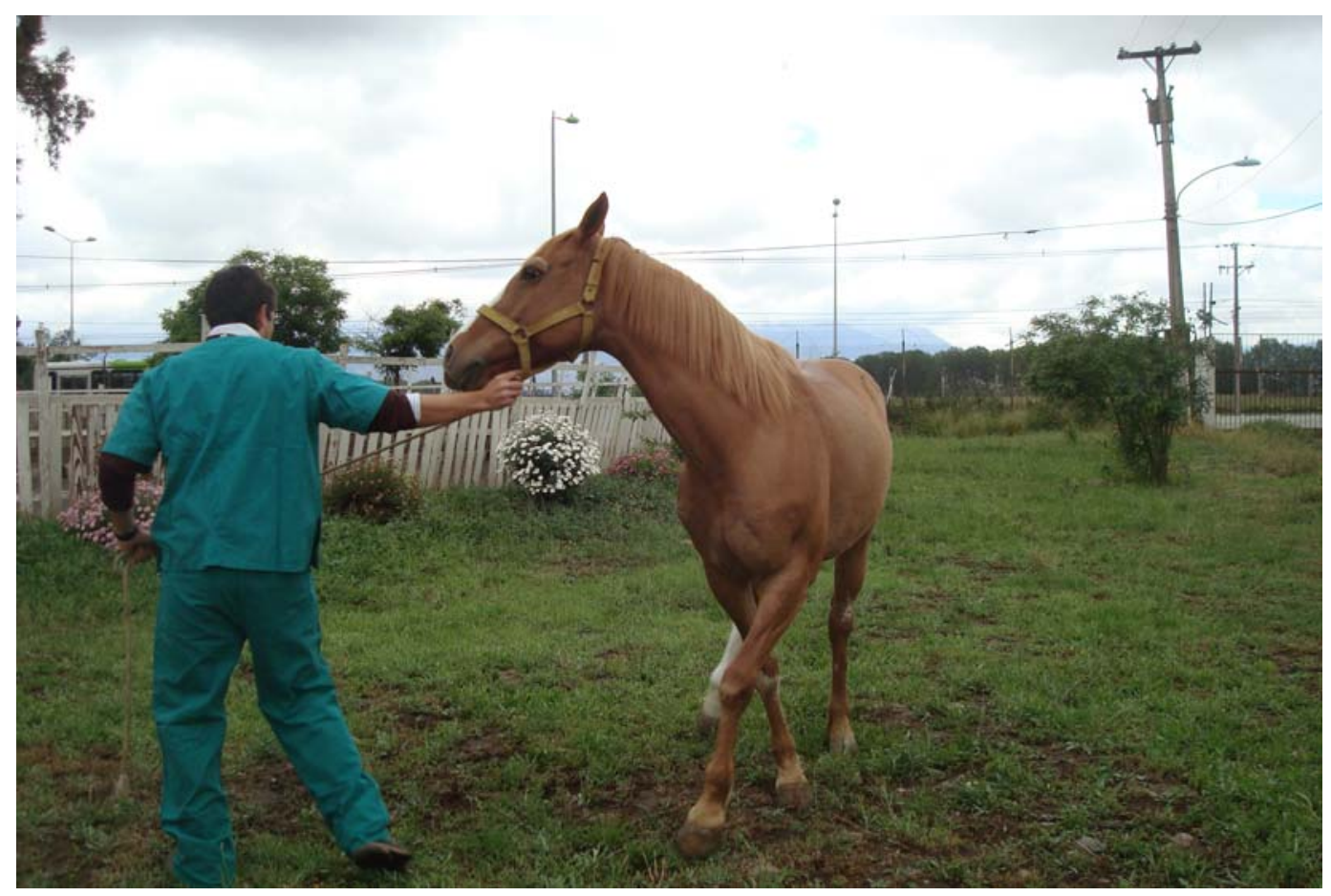

Foto $\mathrm{N}^{\circ}$ 2. Evaluación del caballo en círculos con el objetivo de evaluar la fluidez de los movimientos y/o acentuación de la sintomatología.

Graduación: la graduación va desde 0 a $4+(5+)$. El 1+ una alteración detectable y el 4+ (5+) indica que el paciente se tropieza y posiblemente se cae durante la marcha (Furr y Reed, 2008; Grant, 2010, Mazan, 2010b)

- Grado $0=$ normal

- Grado 1 = Déficit neurológico mínimo que es detectado mediante el uso de pruebas específicas (cruzar miembros, giro en círculo cerrado, cambiar apoyo tirando la cola, caminar en pendiente, etc).

- Grado 2 = Alteración moderada al paso (ejemplar camina como si hubiese sido sedado). La respuesta a las pruebas específicas es obvia.
- Grado 3= Fácil de apreciar al paso. Aparenta como si el animal estuviese borracho al trote. Se hace muy obvio al galope pero el animal no se cae.

- Grado 4 = Muy Atáxico, el caballo se cae al girar en un círculo cerrado o al caminar hacia atrás. Usualmente se rehúsa a andar más rápido que al paso.

- Grado 5 = Decúbito, hasta puede ocurrir que el animal no sea capaz de adquirir la posición de decúbito esternal. 
NEUROLOCALIZACIÓN:

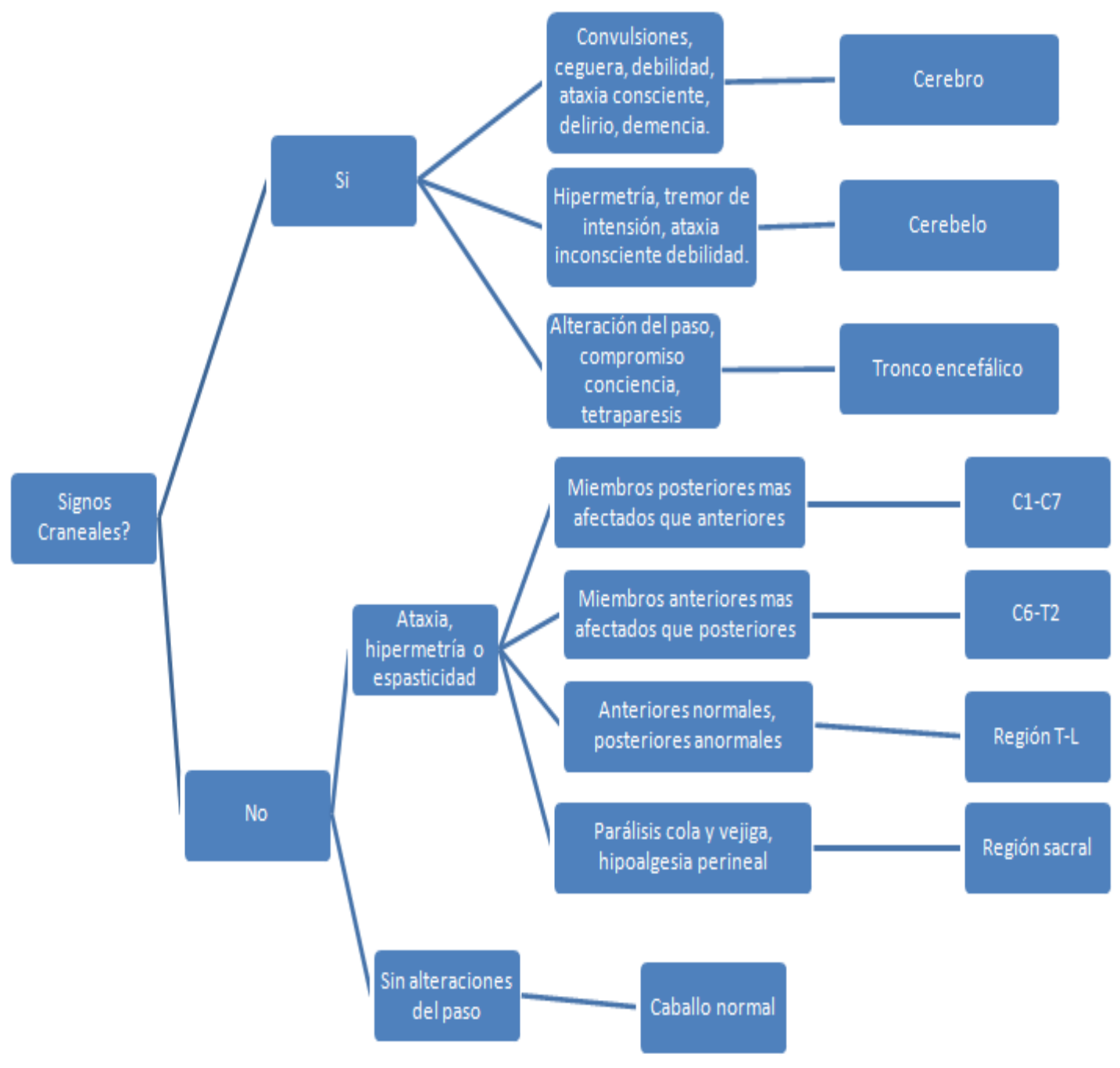

\section{Evaluación del Cuello y de los Miembros Anteriores}

Luego de la evaluación completa de la cabeza y marcha, se procede a evaluar la región del cuello, con lo que se pretende localizar la lesión dentro de $\operatorname{los}$ segmentos $\mathrm{C}_{1}-\mathrm{T}_{2}$.

Se debe realizar inspección y palpación del cuello y miembros para detectar afecciones macroscópicas. El compromiso de neuronas sinápticas pre y post ganglionares causan sudoración local, lo que puede ser de mucha ayuda (Palencia, 2007; Sandoval, 2009). En la rama simpática del sistema nervioso autónomo, el soma (cuerpo celular) de la motoneurona baja preganglionar se ubica en la médula espinal, mientras que los cuerpos celulares de la neurona postganglionar se ubican en un ganglio periférico (Hahn, 2006).

Se debe evaluar la respuesta cervical local y cervicofacial, la que se realiza pinchando suavemente la piel a nivel del cuello lo que va a producir una contracción del músculo cutáneo (cervical local) y también un movimiento hacia rostral de la oreja y contractura de los músculos labiales (cervicofacial) (Foto. $\mathrm{N}^{\circ}$ 3) (Auer y Stick, 2006; Palencia, 2007). La prueba se realiza pinchando la piel a nivel de músculo braquiocefálico comenzado en el principio del cuello (cercano a la cabeza) y continuar hacia el 
hombro Seguir pinchando hacia el miembro para evaluar la respuesta sensorial (Auer y Stick, 2006; Palencia, 2007). La ausencia de este reflejo puede deberse por una alteración del nervio facial, nervios cervicales locales y/o alguna alteración de la medula espinal cervical (Auer y Stick, 2006).

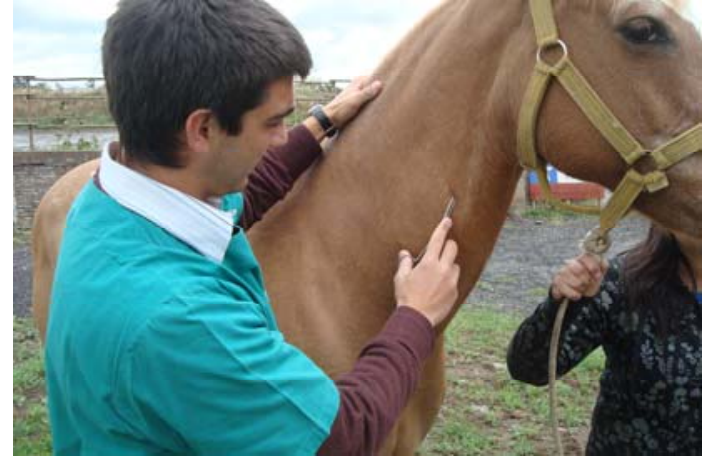

Foto $\mathrm{N}^{\circ} 3$. Evaluación de la respuesta cervicofacial (segmentos C1 - T2).

Test de propiocepción: Esta prueba debe realizarse con el animal en estación y consiste en cruzar las extremidades anteriores, y en ocasiones las extremidades posteriores, cruzándolas sobre la extremidad contralateral (Foto. $\mathrm{N}^{\circ} 4$ ). Una respuesta normal es cuando el animal vuelve el miembro a su posición original de manera inmediata y repone la postura de aplomo en las cuatro extremidades. Se considera anormal, cuando se manifiesta un retraso (y en ocasiones incapacidad) en la reposición de la extremidad. La prueba se suele repetir en varias ocasiones y en la extremidad contralateral, no realizándose habitualmente en casos severos de ataxia por el riesgo de manejo (Palencia, 2007; Grant, 2010; Mazan, 2010, Mazan 2010b).

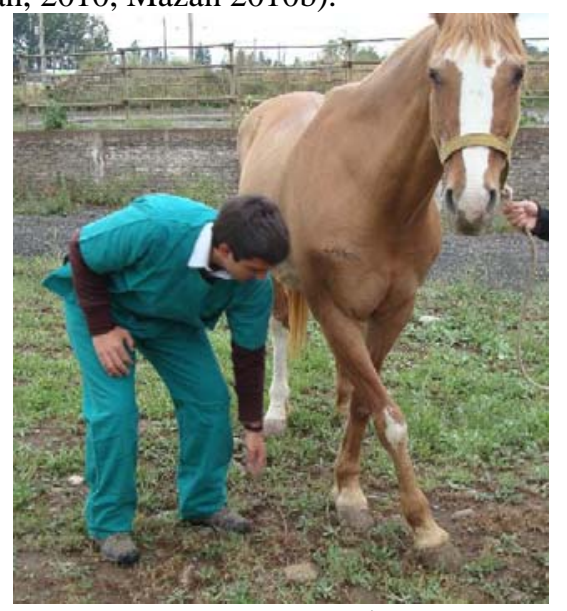

Foto $\mathbf{N}^{\circ}$ 4. Test de propiocepción, cruzamiento de los miembros anteriores
Durante la evaluación pasiva es difícil detectar algún tipo de debilidad de los miembros. En estación y al paso se puede realizar la prueba de “ladeo" o levantar una mano en estación, forzando la que se encuentra en apoyo (Foto. $\mathrm{N}^{\mathrm{o}}$ 5). Estas pruebas buscan evaluar el equilibrio y debilitad del apoyo (Stashak, 2002; Grant, 2010; Mazan, 2010, Mazan 2010b).

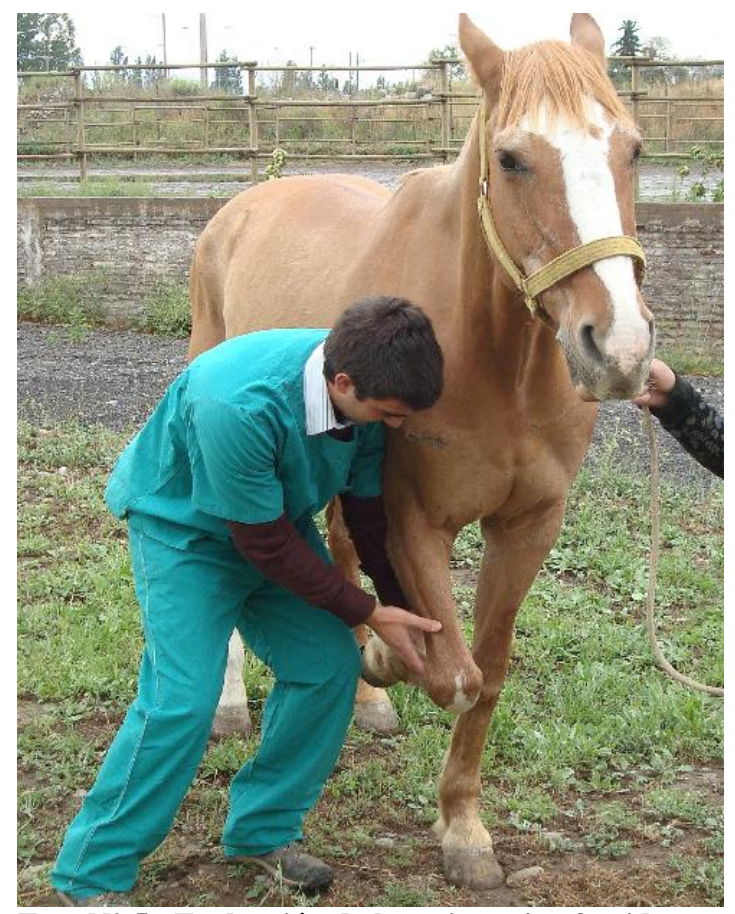

Foto $\mathrm{N}^{\circ} 5$. Evaluación de la resistencia ofrecida por los miembros anteriores mediante la prueba de "ladeo".

Palpación y presión de la cruz. En normalidad se produce una ventroflexión, pero luego aparece resistencia al descenso. En casos patológicos de los miembros torácicos o del dorso, el caballo no ofrece resistencia (Palencia, 2007).

La evaluación del paso debe realizarse en línea recta, alternando el lado por el cual se maneja al caballo. Se debe mantener la cabeza bien firme. Se debe hacer girar al caballo hacia ambos lados (Foto. $\mathrm{N}^{\circ} 2$ ), caminar subiendo o bajando una pendiente con la cabeza en alto o baja en busca de una posible exacerbación de la sintomatología (Foto. No 6) (Auer y Stick, 2006, Palencia, 2007). 


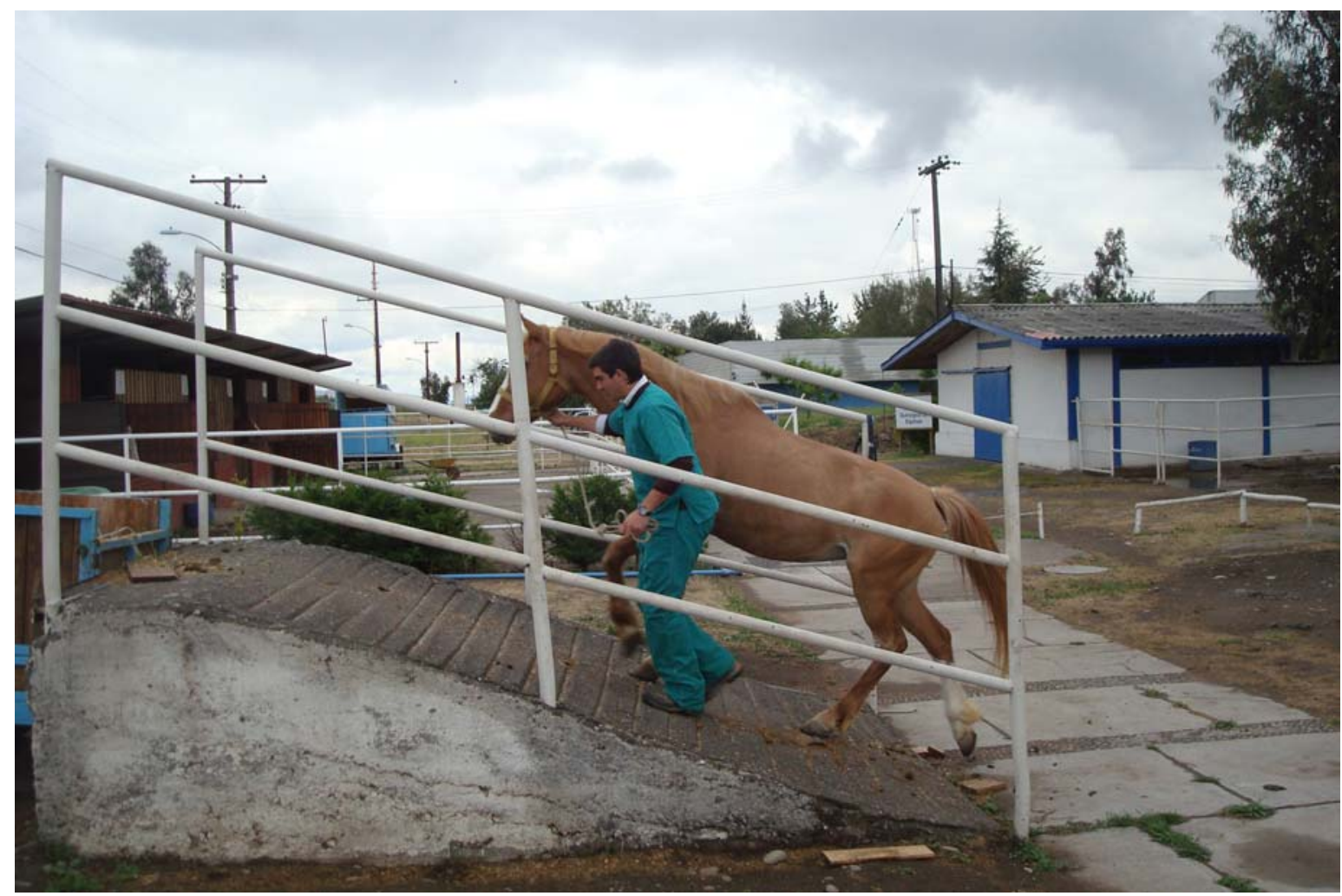

Foto $\mathrm{N}^{\circ}$ 6. Evaluación del caballo en movimiento en una pendiente con la cabeza en alto.

\section{Evaluación del Tronco y de los Miembros Posteriores}

Si solo se evidencian lesiones en el tronco y miembros torácicos, el segmento afectado debiese ser entre $T_{2}-S_{2}$. Se debe inspeccionar el tronco y miembros y palpar cualquier malformación o asimetría. (Auer y Stick, 2006; Furr y Reed, 2008).

La atrofia muscular y sudoración localizada en el tronco o miembros posteriores son de ayuda para localizar la lesión.

Al igual que en el cuello, se pueden realizar pinchazos en el tronco para ver la contracción del músculo cutáneo. Se debe comenzar justo por detrás del hombro y continuar hasta el último espacio intercostal. Para una correcta realización de esta prueba se debe hacer por dorsal y ventral del tronco (Auer y Stick, 2006). Si se afecta esta vía no habrá respuesta del panículo siendo más fácil evaluar en lesiones asimétricas (Auer y Stick, 2006; Furr y Reed, 2008).
La vía refleja comienza en los nervios sensitivos torácicos que viajan al lado ipsilateral de la medula espinal viajando rostralmente, vía interneuronas, para terminar entre los segmentos C8 y L1 de la medula (Auer y Stick, 2006; Furr y Reed, 2008).

Con el ejemplar estático o al paso se puede dar un empujón contra la pelvis o tirar la cola lateralmente con el fin de determinar la resistencia y el movimiento resultante (Foto. $\mathrm{N}^{\circ}$ 7). Un animal débil será fácilmente desplazado (Auer y Stick, 2006).

La evaluación del paso debe realizarse en líneas rectas, alternando el lado por el cual se maneja al caballo. Se debe mantener la cabeza bien firme. Se debe hacer girar al caballo hacia ambos lados, caminar subiendo o bajando una pendiente con la cabeza en alto o baja en busca de una posible exacerbación de la sintomatología (Foto. $\mathrm{N}^{\circ}$ 6) (Auer y Stick, 2006, Palencia, 2007). 


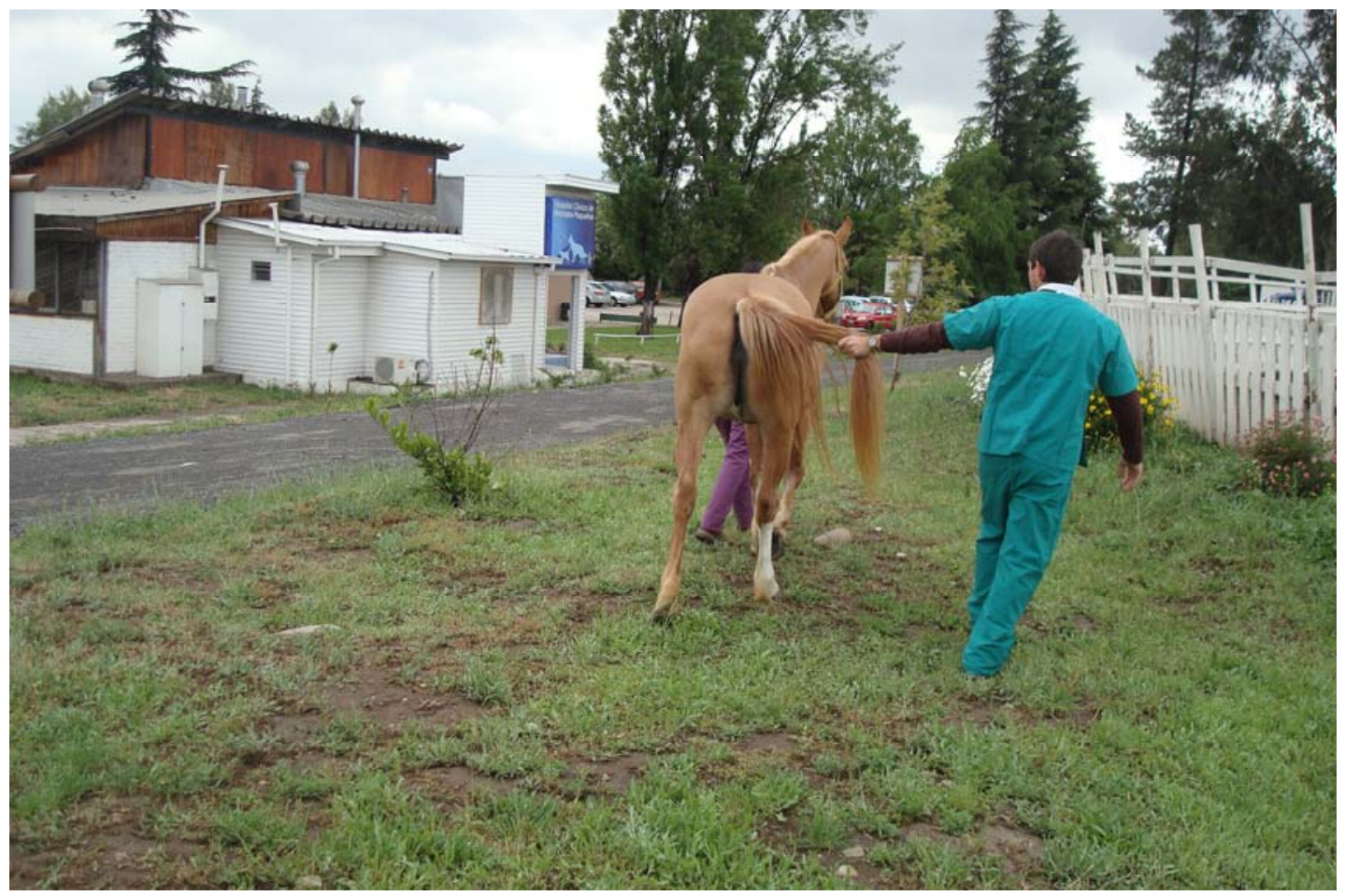

Foto $N^{\circ} 7$. Evaluación de la resistencia ofrecida por los miembros posteriores al tirar la cola lateralmente.

\section{Evaluación de la Cola y del Ano}

Se evalúan los segmentos medulares sacros y coccígeos. Este segmento es el indicado evaluar si existe una sospecha del síndrome de cauda equina (Dörner, 2009). La cauda equina se define por la medula espinal y nervios que se encuentran caudales al segundo segmento sacral de la medula espinal. Debido a la incongruencia entre los segmentos de la médula espinal y de los segmentos vertebrales, la cauda equina está caudal a la unión lumbosacra (Furr y Reed, 2008).

En esta etapa final del examen se debe evaluar (Grant, 2010; Mazan, 2010, Mazan 2010b):

- El tono de la cola: Evaluar si se encuentra flácida 0 si existen movimientos voluntarios.

- Los reflejos perineales: Leve pellizcamiento del periné (Foto. $\mathrm{N}^{\circ} 8$ ) observando una contracción refleja del esfínter anal y de la flexión de la cola. Las ramas sensitivas provienen del nervio pudendo $\left(\mathrm{S}_{1}-\mathrm{S}_{3}\right)$ y la contracción está dada por la rama rectal del nervio pudendo y la flexión de la cola por los segmentos medulares y coccígeos $\left(\mathrm{S}_{1^{-}}\right.$ Co).

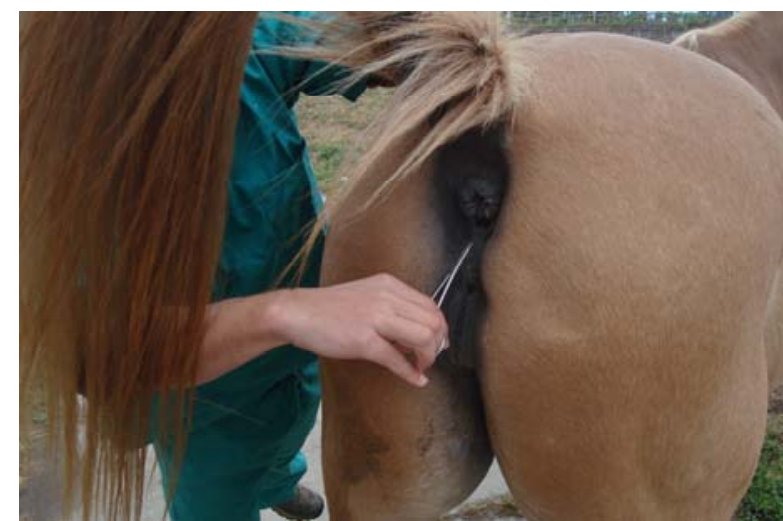

Foto $\mathbf{N}^{\circ}$ 8. Reflejo perianal. Evaluación de la región sacral.

En definitiva una lesión a este nivel va a producir: hipoalgesia, hiporreflexia, hipotonía y atrofia muscular de la cola, esfínter anal, periné, caderas y cara caudal de los muslos (Reed et al, 2004).

\section{Evaluación del Caballo en Decúbito}

Cuando nos encontramos frente a un caballo en decúbito debemos realizar las pruebas necesarias para neurolocalizar la lesión. Se debe pesquisar si es que el caballo presenta o no movimientos voluntarios (Auer y Stick). 
- Estado de consciencia.

- Reflejos espinales: Reflejo cervicofacial, reflejo del panículo.

- Sensibilidad cutánea: Miembros, torso.

- Función de los miembros: Evaluar el tono extensor mediante la resistencia a la flexión pasiva del miembro. Realizar la prueba de flexión pellizcando la piel: una respuesta negativa se confirma cuando el reflejo está disminuido o ausente y puede ir acompañado por una extensión refleja de la mano o pata contralateral (reflejo extensor cruzado)

- Reflejo patelar: sostener la pata del animal con una moderada flexión y golpear el ligamento patelar medio. Una respuesta positiva se evidencia con extensión de la pata.

- $\quad$ Reflejo Tibial, reflejo del gastrocnemio y reflejo tibial craneal: Se debe golpear detrás del trocánter mayor, tendón de Aquiles cercano a su inserción y en el cuerpo del músculo tibial craneal, respectivamente.

- Reflejo del tríceps, reflejo bicipital, reflejo carpo radial: Para el reflejo tricipital se debe tener la mano flectada y se golpea el tríceps esperando una extensión del codo. Para el caso del reflejo bicipital se debe golpear la parte delantera del codo y se debe producir la extensión del hombro y flexión del codo. Para el último, se debe golpear el cuerpo del músculo carpo radial y observar la extensión del carpo.

\section{Conclusiones}

En el caballo de deporte en nuestro medio, la frecuencia de presentación de afecciones del sistema nervioso por causas sistémicas, infecciosas y/o de carácter metabólico son bajas. Sin embargo al considerar el trauma como factor etiológico la prevalencia aumenta significativamente, especialmente en ejemplares sometidos a exigencias deportivas mayores como velocidad, salto y polo.
Una aproximación sistemática y acuciosa del sistema nervioso en el caballo es la única forma de realizar una evaluación correcta del ejemplar y llegar a buen término frente a una eventual neuropatía.

El diagnostico correcto de una neuropatía requiere por parte del profesional un conocimiento acabado de aspectos relacionados con neuro fisioanatomía $\mathrm{y}$ las enfermedades que afectan al sistema nervioso en esta especie.

El examen neurológico debe incluir la evaluación completa del ejemplar, la cabeza, cuello, torso, miembros anteriores y miembros posteriores mientras el animal se encuentra en estación o en movimiento. Además realizar un completo examen de los otros sistemas del ejemplar.

Se debe considerar que algunos exámenes que se podrían realizar en el caballo y que serían de gran utilidad, muchas veces son impracticables con el animal en pie dada las características propias de esta especie, como por ejemplo evaluar los reflejos de los miembros.

\section{Referencias}

1. Auer, J.; Stick, J. 2006. Equine Surgery. Tercera Edición. USA. Editorial Saunders. Pp. 642-659.

2. Budras, Kd.; Sack, W.; Röck, S. 2003 . Anatomy of the Horse: An Illustrated Text. Cuarta Edición. Franckfurt, Alemania. Editorial Schlütersche $\mathrm{GmbH}$ and Co. 152 p.

3. Colahan, P.; Mayhew, I.; Merritt, A.; Moore, J. 1998. Medicina y Cirugía Equina: Vol. I. Cuarta edición. Buenos Aires. Argentina. Editorial Intermédica. $1736 \mathrm{p}$.

4. Dörner, C. 2009. Sindrome Cauda Equina. Informe Diplomado Medicina Equina. Santiago, Chile. U. Chile Fac. Cs. Veterinarias y Pecuarias. $20 \mathrm{p}$.

5. Furr, M,; Reed, S. 2008. Equine Neurology. Primera Edición. USA. Editorial Blackwell Publishing. 412 p. 
6. Grant, B. 2010. Neurological Examination: Diagnosis for Neurological Problems. [en linea] $<$ http://www.equinewobblers.com/Diagn ostics/Neurological_Examination.htm> [consulta 27/10/2010]

7. Guyton, A.C., Hall, J.E. 2001. Tratado de Fisiología Médica. Décima Edición. Editorial Mcgraw Hill Interamericana. México. 1280 p.

8. Hahn, B. 2006. Miscellaneous disorders of the equine nervous system: Horner's Syndrome and polyneuritis equi. Clin Tech Equine Pract 5: 43 - 48.

9. Mackay, R.; Mayhew, I. 1998. Enfermedades del Sistema Nervioso. En: Medicina y Cirugía Equina: Vol. I. Cuarta edición. Buenos Aires. Argentina. Editorial Intermédica. 1736 p.

10. Mackay, R. 2006. Diagnostic Procedures. En: Equine Surgery. Tercera Edición. USA. Editorial Saunders. 1455 p.

11. Mackay, R. 2010a. The Art and Science of Equine Neurology - The Spinal Cord. Proceedings of the AAEP Annual Resort Symposium. Guanacaste, Costa Rica. 8996.

12. Mackay, R. 2010b. The Art and Science of Equine Neurology - Peripheral Nerves \& Stringhalt. Proceedings of the AAEP Annual Resort Symposium. Guanacaste, Costa Rica. 96-107.

13. Mackay, R. 2010c. The Art and Science of Equine Neurology - The Forebrain. Proceedings of the AAEP Annual Resort Symposium. Guanacaste, Costa Rica. 108-113.

14. Mackay, R. 2010d. The Art and Science of Equine Neurology - The Brainstem \& Cranial Nerves. Proceedings of the AAEP Annual Resort Symposium. Guanacaste, Costa Rica. 114-120.

15. Masty, J. 2008. Overview of Neuroanatomy. En: FURR, M. y REED, S. Equine neurology. $1^{\text {a }}$ ed. USA, Blackwell Publishing. pp. 3 - 31 .
16. Mayhew, J. 2008. Equine Neurology: Watching Horses Strangely. Proceedings of the 47th British Equine Veterinary Association Congress. Liverpool, United Kingdom.

17. Mayhew, J. 2009a. Emerging Equine Neurologic Syndromes: What nature taught us recently about Neurophysiology and Neuroanatomy. Proceedings of the 11th International Congress of World Equine Veterinary Association. Guarujá, SP, Brazil.

18. Mayhew, J. 2009b. Equine Neurologic Examination - What do I really Look for?. Proceedings of the 11th International Congress of World Equine Veterinary Association. Guarujá, SP, Brazil.

19. Mazan, M. 2010. The Neurological Examination - Part I. Tufts Cummings School of Veterinary Medicine. [en linea]

<http://www.tufts.edu/vet/sports/neuro1. html> [consulta: 29/10/2010].

20. Mazan, M. 2010b. The Neurological Examination - Part II. Tufts Cummings School of Veterinary Medicine. [en linea]

<http://www.tufts.edu/vet/sports/neuro2.

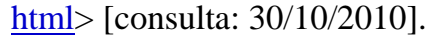

21. Orsini, J.; Divers, T. 2000. Manual de urgencias en la clínica equina: Tratamientos y técnicas. España. Editorial Elsevier. 745 p.

22. Palencia, P. 2007. Medicina Interna Equina Neurología Equina; el examen clínico del caballo. Cantabria. España. Información veterinaria: 16-20.

23. Radostits, O.; Mayhew, I.; Houston, D. 2002. Examen y diagnostico clínico en veterinaria. Primera Edición. Madrid, España. Editorial Harcourt-Saunders. 757 p. 
24. Reed, S., Bayly, W., Sellon, D. 2004. Equine Internal Medicine. Segunda edición. USA. Editorial Elsevier. 1658 p.

25. Sandoval, C. 2009. Sindrome de Horner. Informe Diplomado Medicina Equina. Santiago, Chile. U. Chile Fac. Cs. Veterinarias y Pecuarias. $12 \mathrm{p}$.

26. Stashak, T.S. 2002. Adam`s Lameness in Horses. Quinta Edición. Philadelphia. U.S.A. Editorial Lippincott Williams and Wilkins. 1200 p. 